\title{
Demystifying Occupational Stress and Fatigue Through the Creation of an Adaptive End-User Profiling System
}

\author{
Bruce I. Reiner • Elizabeth Krupinski
}

Published online: 6 December 2011

(C) Society for Imaging Informatics in Medicine 2011

\section{Introduction}

Occupational stress and fatigue are commonly encountered challenges in medicine and have been shown to adversely affect quality and patient safety [1-3]. As medicine continues to adapt to changes in health-care economics, technology, and expectations for service deliverables, the potential for occupational stress and fatigue steadily increases [4, 5]. Due to its insidious nature, occupational stress and fatigue often go undetected until a catastrophic event takes place, mandating a thorough review of the underlying cause (i.e., root cause analysis). This analysis often identifies such human errors as misdiagnosis, poor communication, and/or incomplete/delayed data analysis. A myriad of precipitating factors can contribute to these faulty actions including excessive workload, limitations in data accessibility, sleep deprivation, and suboptimal use of technology, all of which can contribute to occupational stress and fatigue.

Medical imaging is especially susceptible to occupational stress and fatigue which can take a myriad of forms including visual and decision fatigue. Visual fatigue is the result of prolonged human-computer interaction, which can result in eye strain and faulty image perception and interpretation [6, 7]. Decision fatigue is the result of lengthy and intense

B. I. Reiner $(\bowtie)$

Department of Radiology,

Veterans Affairs Maryland Healthcare System,

10 North Greene Street,

Baltimore, MD 21201, USA

e-mail: breiner1@comcast.net

E. Krupinski

Department of Radiology, Psychology and the Arizona

Telemedicine Program, University of Arizona,

1609 N Warren, Bldg 211, Rm 112,

Tucson, AZ 85724, USA workload, which can result in misdiagnosis, diagnostic uncertainty, and inefficient workflow [8-10]. The various forms of occupational fatigue have been shown to be contributing factors in preventable medical errors, which undermine the credibility of both individual and institutional service providers, can result in costly medical malpractice lawsuits, and lead to adverse clinical outcomes.

In order to better understand and address the various causes of occupational stress and fatigue, objective data collection and analysis is an imperative. A number of technologies and tools currently exist that quantify various measures of stress and fatigue. At the same time, the analysis must take into account that occupational stress and fatigue are dynamic processes, with marked inter- and intra-observer variability. Quantifying and understanding this end-user variability is essential for creating an effective means for better understanding the cause and effect relationship between occupational fatigue and adverse clinical outcomes.

\section{Creating a Customizable Profiling System}

The concept of end-user profiling in medical imaging has been described in a number of clinical applications and is designed to take into account the uniqueness of each individual end user [11, 12]. Medical imaging professionals (e.g., radiologists, technologists) differ in a variety of ways, including clinical experience, education and training, demographics (e.g., age, gender), and specific job requirements. In addition to these more obvious sources of variability, other (often unnoticed) factors contribute to inter-observer variability including technology proclivity, personality [13], intelligence, emotional state, and sensory/motor skills (Table 1). All of these variables contribute in varying degrees to the collective 
Table 1 Profile components for medical imaging professionals

1. Demographics (e.g., age, gender)

2. Education and training

3. Clinical experience

4. Occupational setting

5. Intelligence

6. Personality

7. Emotional state

8. Computer experience and proclivity

9. Sensory and motor skills (including image perception)

attributes and characteristics of individual end users and should be considered when attempting to understand and quantify occupational stress and fatigue.

Another important factor to consider in end-user fatigue profiling is that occupational stress/fatigue is a dynamic process that can change over time. An external event (e.g., divorce, illness) may serve as a source of emotional turmoil and dramatically alter an individual's coping skills and ensuing stress levels. As an example, a radiologist may experience increased fatigue over the course of a single working shift that may be influenced by changes in exam volume and complexity (i.e., cognitive overload). Differences in sleep may in turn influence the degree of fatigue variability in a single radiologist, with higher-quality sleep resulting in less intra-day fatigue variability than poorer quality sleep. At the same time, two different radiologists may experience different degrees of intra-day fatigue variability (given the same sleep quality and workload), as a function of differences in their emotional state or personality.

The net result is that radiologists (or technologists) are not a homogenous population. In order to account for these differences and create effective intervention strategies, customizable end-user profiling is essential. In order to completely account for factors with the potential to influence occupational stress and fatigue, additional characteristics of the workplace and technology in use should be considered (Table 2). The profiling system should be designed to take into account all intrinsic and extrinsic factors which affect individual performance and fatigue and provide a quantifiable mechanism to define interaction effects and confounding variables.

\section{Real-Time Customizable Data Analysis}

In order to accurately determine the degree, impact, and interaction effects of occupational fatigue on performance, a number of data elements must be recorded and analyzed (Table 2). Each individual data category is designed to capture data which can be directly correlated with objective

fatigue measures and determine the relationship between that data category and the individual end user and task being performed. For task performance, it is essential that an objective methodology be utilized to quantify the overall complexity (i.e., level of difficulty) for the task being performed, which in turn can be correlated with the specific attributes (e.g., education/training) of the individual end user. A methodology for objectively quantifying imaging exam complexity has been reported [14], with specific applications relevant to individual radiologist workflow [12, 15].

In addition to individual task complexity, the cumulative workload and duration of the working shift are important data in analyzing occupational fatigue. One can reasonably assume that fatigue accumulates over the duration of the working shift and is exacerbated by progressively increasing workload, which produces cognitive overload and decision fatigue. A number of additional factors can be included in the analysis, such as frequency and duration of resting periods (i.e., breaks), which can influence cumulative fatigue given the same workload and shift length. An individual end user's personal experience and familiarity with the task being performed are also important, for one would assume that greater experience would generally correlate with decreased fatigue (or more likely the development of strategies to recognize and counteract fatigue). The quality and quantity

Table 2 Classification of data for occupational stress/fatigue analytics

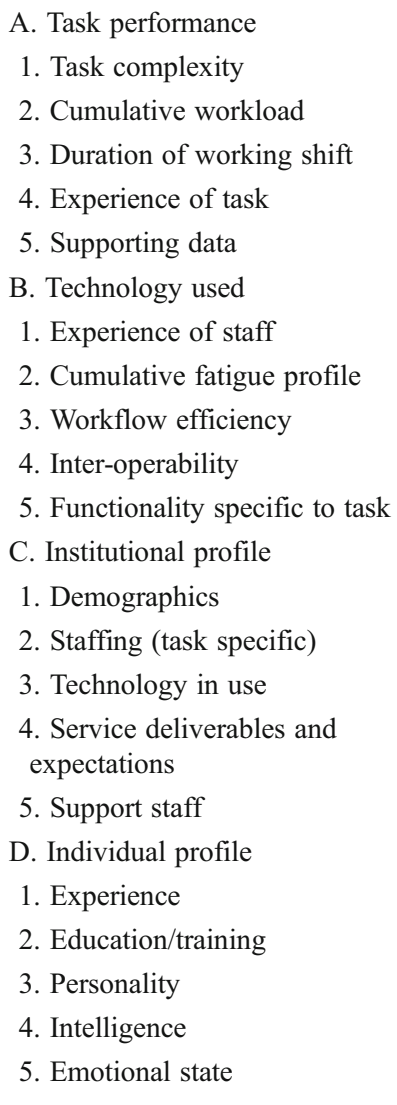


of clinical data accessible to the interpreting radiologist can affect diagnostic confidence and report accuracy, which in turn may influence fatigue $[16,17]$.

Technology is of particular importance in assessing occupational fatigue, due to the dependence of radiology practice on imaging and information system technologies [8]. Unfortunately, not all end users embrace and utilize new technologies as efficiently as their peers. In fact, the implementation of new technologies can in itself serve as a precipitant for occupational stress/fatigue, which is highly dependent upon each individual end user's openness and receptivity to innovation and change [18]. In addition to this individualized fatigue response to technology, each individual technology has its own cumulative "fatigue profile." While one would expect a distribution of individual fatigue scores for a given technology (e.g., electronic medical record) within a given institution, if one compared different technology options across multiple institutional and individual end users, one could create a cumulative fatigue profile for that technology, relative to its peer technologies.

In addition to these "macro" measures of technology, additional "micro" measures related to technology are important in analyzing occupational fatigue. The specific functionality, workflow, and inter-operability of a given technology (specific to a particular task) should be analyzed to determine its role in affecting fatigue. From an operability standpoint, two individual technologies (e.g., picture archiving and communication system and EMR) may have low fatigue profiles in isolation, but when used in tandem may be associated with high levels of fatigue due to poor inter-operability. A radiologist tasked with the interpretation of a complex imaging dataset on a PACS may require access to clinical data on the EMR. If this requires the radiologist to open up a separate application to retrieve these data, then workflow is impaired and fatigue levels may rise. The analysis of fatigue data requires both straightforward (i.e., single variable) as well as complex (i.e., multivariate) analyses.

A fatigue profile for an institution should also be considered. On a simplistic level, a radiologist working in a highly efficient outpatient imaging center would be expected to experience lower levels of occupational stress/fatigue than his/her counterpart in a large urban tertiary care hospital, given the same workload and task complexity. A number of variables may account for these differences in fatigue, including environment, staffing, technology, workflow, and politics. Something as simple as decreasing the number of interruptions (by clinicians, clerical staff, or technologists) can have a profound impact on cumulative productivity and fatigue [19]. The purpose of the "fatigue profile" is to accurately identify these contributing factors, prospectively record data throughout the working shift, and analyze these data to identify trends specific to the individual and collective population.

\section{Customizable Feedback and Education}

The creation of a standardized fatigue database provides the opportunity to perform analyses which can be customized to the specific needs and preferences of end users. In addition to the radiologist or technologist who is the primary source of analysis, other interested parties include the radiology department chief, hospital administrator, IT/PACS administrator, and technology vendor. The derived analyses are not intended to be punitive, but instead serve as information sources to educate, improve workflow, staffing, technology procurement and upgrades, and workload distribution.

In the example of the radiologist whose primary task is interpretation of medical imaging exams, he/she may be interested in the following analyses:

1. How do fatigue measures typically change over the course of the working shift?

2. What specific tasks correlate with higher fatigue measures?

3. Are there specific days/times associated with peak fatigue levels?

4. How does fatigue change with the introduction or upgrade of a given technology (e.g., PACS, reporting system, CAD)?

5. Do higher levels of fatigue correlate with quality deficiencies?

6. How do my individual fatigue levels and variability relate to those of my departmental colleagues performing similar tasks and workloads?

7. What specific factors (e.g., sleep quality, caffeine intake) affect my individual fatigue measures over time?

8. How can I better utilize existing technology to improve performance and reduce fatigue?

Slightly different perspectives and analyses would be applicable to other parties. The radiology department chief who oversees the collective performance of all radiologists would be interested in determining the relationship between fatigue and performance (e.g., productivity and quality metrics) on both collective and individual levels. For those radiologists who demonstrate fatigue/performance measures significantly above or below the rest of the group (i.e., outliers), the chief would want to identify overall trends that could explain these differences and seek out solutions for positive change. As an example, the chief may determine that one particular neuroradiologist has higher and more consistent quality performance metrics than his/her peers for relatively similar fatigue levels and workloads. Through more detailed analysis of workflow, technology usage, and continuing medical education, he/she may gain insight to improve fatigue/performance of other neuroradiologists with lower fatigue/performance measures. A similar analysis would be applicable to a radiology or hospital administrator whose primary focus would be on 
fatigue/performance of technologists and the technology in use.

Each individual end user could readily create customized analytics based upon individual preferences and needs. These customizable analytics could be delivered in the time, manner, and content of individual choosing. In addition to regularly scheduled analytics, automated prompts could be delivered when certain pre-determined thresholds are reached. As an example, a technologist may request that an automated prompt be delivered to his/her work console in the event that a pre-determined fatigue measure is recorded and use this for scheduling breaks or requesting assistance from support staff. Another example may be the radiologist who requests that the system notify him/her when specific tasks associated with high historic fatigue levels are assigned to his/her workload. In this example, a general teleradiologist may be assigned a chest CT angiography for the evaluation of pulmonary emboli. Based upon computerized analysis of exam complexity and the specific radiologist's historic performance/fatigue data, the exam is determined to be associated with a "high fatigue risk." The radiologist could utilize this prompt to modify workflow, modify technology usage (e.g., CAD), request consultation from a peer, or request that the exam be reassigned.

A technology vendor or prospective customer could utilize these data to identify fatigue/performance trends specific to the technology and user group. By utilizing the data from the various profile groups, a vendor or customer could determine the relative strengths and weaknesses of a certain technology for a given task and perform a comparative analysis of competitive technologies. As an example, a radiology administrator interested in purchasing a new PACS wishes to determine which vendor's product would be best suited for his/her department. By utilizing data from the fatigue database institutional profile, he/she could request an analysis of those institutions that closely match the profile of his/her host institution. The administrator could then compare different vendors' PACS based upon specific search criteria deemed to be of highest priority. A technology vendor could utilize the database in a similar fashion to determine the relative strengths/deficiencies of their product relative to competitors or specific to task performance and institutional/individual profiles. These data could in turn assist the vendor in prioritizing $\mathrm{R} \& \mathrm{D}$ efforts aimed at performance improvement of the technology in question. In the end, the standardized fatigue database becomes an empowerment tool for customable feedback and education; which can facilitate performance improvement and occupational stress/fatigue reduction. Knowledge of existing deficiencies can guide education and training for individual and collective end users, while providing before and after analyses to determine the relative impact of the educational effort on performance.

\section{Conclusion}

The creation of a standardized database which tracks fatigue and performance metrics could provide an objective and reproducible methodology for understanding the complex relationship between occupational fatigue and performance. The value of the analyses is in part dependent upon the ability to analyze data specific to the individual needs, preferences, and profile of the end user. The creation of profiling groups provides an objective mechanism for comparative analysis of "similar users," taking into account individual/institutional characteristics and attributes, technology in use, and tasks being performed. The ultimate goal of these analytics is the reduction of medical errors through customizable feedback and education, aimed at reducing fatigue and improving clinical performance.

\section{References}

1. Leape LL: Errors in medicine. JAMA 272:1851-1857, 1994

2. Kohn LT, Corrigan J, Donaldson MS: To err is human: building a safer health system. National Academy Press, Washington DC, 2000

3. Helmreich RL: On error management: lessons from aviation. BMJ 320:781-785, 2000

4. Burbeck R, Coomber S, Robinson SM, et al: Occupational stress in consultants in accident and emergency medicine: a national survey of levels of stress at work. Emerg Med J 19:234-238, 2002

5. Piko B: Work-related stress among nurses: a challenge for healthcare institutions. Perspect Public Health 119:156-162, 1999

6. Krupinski EA: Medical image perception issues for PACS deployment. Semin Roentgenol 38:231-243, 2003

7. Krupinski EA, Berbaum KS, Caldwell RT, et al: Long radiology workdays reduce detection and accommodation accuracy. J Am Coll Radiol 7:698-704, 2010

8. Reiner B, Krupinski EA: The insidious problem of fatigue in medical imaging. J Am Coll Radiol (in press), 2011

9. http://www.nytimes.com/2011/08/21/magazine/do-you-suffer-fromdecision-fatigue. Accessed Sept. 28, 2011

10. Baumeister RF: The psychology of irrationality. In: Brocase I, Carrillo JD Eds. The Psychology of Economic Decisions: Rationality and Well-Being. Oxford University Press, Oxford, 2003, pp 1-15

11. Reiner B: One size (doesn't) fit all. J Am Coll Radiol 4:567-570, 2008

12. Reiner B: New strategies for medical data mining. Part 2: the customizable productivity pacer. J Am Coll Radiol 8:33-38, 2011

13. Matthews G, Emo AK, Funke G, et al: Emotional intelligence, personality, and task-induced stress. J Exp Psychol 12:96-107, 2006

14. Reiner B: New strategies for medical data mining. Part1: accountable and transparent reimbursement. J Am Coll Radiol 7:975-979, 2010 
15. Reiner B: New strategies for medical data mining. Part 3: automated workflow and optimization. J Am Coll Radiol 8:132-138, 2011

16. Reiner BI: Medical imaging data reconciliation. Part 1: innovation opportunity. J Am Coll Radiol 9:622-625, 2011

17. Reiner BI: Medical imaging data reconciliation. Part 2: clinical order entry/imaging report data reconciliation. J Am Coll Radiol 10:720-724, 2011
18. Reiner BI: Optimizing technology development and adoption in medical imaging using the principles of innovation diffusion. Part 1: theoretical, historical, and contemporary considerations. J Digit Imaging 5:750-753, 2011

19. Reiner B, Siegel E, Protopapas Z, et al: Impact of filmless radiology on the frequency of clinician consultations with radiologists. AJR 173:1169-1172, 1999 